\title{
解析电力系统及其自动化技术
}

叶露

广西容县电力有限公司

DOI:10.32629/hwr.v2i12.1776

[摘 要] 本文主要说明了电力体系的一些现代化技术,其中包含: 实际工作的总线控制配置、光传输并运用两种以上的处理 的计算方式技术、不同于传统对象数据库的被动形式而产生的主动式技术,在这里都做了一一的说明; 之后对于电力体系现 代化的一些相关技术的运用进行了深刻的叙述, 以此希望能给相关人士一些有利的参照。

[关键词] 电力体系; 自动化; 电力系统

目前电力在现实中的运用可以说越来越现代化、条理 化, 对于我们日常生活来说, 对电力的依赖越来越多, 而电 力技术的使用可以说同社会的进步是紧紧相连的。所以, 要为电力体系和一些现代化技术提一个定义的话。可以将 电力体系定义为: 由对电的引发、变化、传输、配置、使 用等等的一些有关模式构成, 并运用产生电力的设备, 将大 自然中的天然资源变成电力资源, 然后运用变化和配置电 力的系统将电能运送到负荷中心, 再将其投入到一些相关 的设施上, 转变成生活能源, 并为人们的日常生活供应方 便。

\section{1 电力体系和现代化技术说明}

1.1 实际工作中的总线掌控系统

对于工作中的总线的掌控系统指的就是在装配的时候, 将一些自动化配置, 如: 自动仪表, 将其同相关的控制设施 进行相连, 并产生一种非单向的网络数据化。一般来说实际 工作中的总线技术会拥有运算及通讯功用, 且采用掌控仪 表相互之间产生的脉络体系, 并对工作中的相关数据内容 进行控制, 其通过工作本身的需求对于讯息和相关内容实 施现代化掌控。工作中的总线控制技术可以说是一种敞开 且分散的控制体系, 它使用了网络化的控制体系, 且完成了 对于系数、控制、预警、实际呈现的全方位现代化功用。 就现在来看, 在国内使用的最多的就是分散式的控制体系。

1.2 主动的对象数据库技术

主动的对象数据库技术广泛应用于电力系统的监视与 控制过程中, 对于系统的开发与设计也有着直接的影响。, 目前就电力体系在监控的时候应用得最为广泛, 并对相关 的体系的研究创新及新理念的产生也有着一定作用。这种 技术方式同传统技术比较可以说更具有主动性及相关的技 术性, 该技术能够将系统内部相关数据进行评判以及思考, 并对其存储库中的对应数值进行掌控, 并让数据本身的统 一平稳有所增强。

\section{3 运用光同时进行处理的技术}

对于电力体系现代化保护有一种举措那就是使用光来 同时进行处理的技术, 可以说光的传输拥有十分大的灵巧 特质, 并且电容负荷并不会对其产生作用。

\section{2 电力体系创立模型之后的共享实力}

电力体系现代化发展十分迅速, 而在其发展中, 其体系 通常都表现在对于地與空间特性的叙述上面, 并且以空间 结构为主要特点进行模仿地與体系的想法已经差不多是一 种规范, 可是在现实里, 对于它的掌控对象会有着十分繁复 的电力规律体系构成。因此创立电力体系独有的空间思考 研究模型是很有用的。对于这样的针对语义层次的资源共 有, 最本质的需求就是供给和所求的两者要对相同的资源 数据看法相同, 因为相同的认识才能确保这一点的顺利进 行, 所以在资源分享的时候要有一个电力体系的最初的维 形, 并以此来作为部门之间资源分享的基本点。其中它包 含:（1）地與实际的几何特性的准确意义及表述, 包括电力 体系对于服务应用所包含的空间方位结构特性; (2) 对于物 体的规律特性的基础意义和表示, 在电力体系上看, 它有着 物理构架, 每一个组成部分和大体的物理特性、运行方面的 一些讯息分享、全面多方位、动态的一些运用和思考。

\section{3 电力体系集中度增高}

对于电力体系的产生和进展过程要从市场经济的需求 动力来看, 不管是该体系的产生是创立在相关共通的还是 专门电力体系现代化平面上, 对于多范围多层次的适用判 断及高效率的经营需求, 都需要更合乎标准的资源共有、全 面多元化的使用研究思考。所以, 要将一些从前的信息方式 推翻, 并采用数据资源和运用的整体结合, 并且将多面化多 方位的空间讯息同多样的有关讯息进行整合相连,并将空 间的运算同主流运算相结合, 全方位的展现资源之间的相 互联系, 是今后电力体系现代化进展的必然方向。

\section{1 电力体系现代化、讯息化}

因为使用者所面临的数据操作性非常的强, 因此, 为了 能让使用者更好的进行掌握及运用, 加强数据本身的易掌 握性是很重要的。除了这点以外, 还要给予相应的图形标志, 并让其标志可以很形象的同相关对象对应, 允许地理及非 地理两类图形的相结合整体管制功效。对于电力体系的现 代化应用的实施性需求也十分的高, 运用多种对象的后期 捆绑技术, 一个对象所归属的分类可以在工作的时候进行 限定, 而不是已经成为目标码以后再进行肯定。所以, 使用 
者能在目前所拥有的笼统数据及空间运作包上面, 任何时 候对本身所要的数据的分类及工作方式进行定位, 以此来 加强系统本身的开创性及扩展性。进行电力体系现代化的 目标就是完成企业规范化、讯息化。像这样的集很多技术 和讯息为一个整体的企业级讯息体系, 从经济和可实施的 视角上来说极需要一个集思考策划并一步步的进行实施的 总体计划。

\section{2 完善数据库}

使用不同的数据库对数据进行存放和管制, 而对于它 的数据复制及安全性能是别的文件管制方式所比不了的。 就现在看, 新创立的系统拥有许多优点, 因为其体系以关系 数据库管制体系为主要应用, 并运用它较强的监管能力及 能进行多集检索的方法, 能十分有利的让网络负荷下降, 并 快速的将要查的方向进行实指性定位, 当出现几位使用者 同一时间进行探访时, 时效就会大大的加强了。虽然这个系 统存在着许多优点, 但其缺点也是不容忽视的, 因为在电力 体系中, 对于空间的相关数值的长度是可以变化的, 所以对 于其繁复的空间就要添加一些所对应的相应功能; 对于这 点, 它将很难达成对于空间相应数值的一些图形功用及基 础工作; 而且对于空间对应该的相应顺序很难进行叙述。 随着科技的进步, 对于相应的数值管制体系及相应关系数 值管制体系进入了一种商业化走向, 因为它们属于一种可 以进行扩充的数据库管制体系, 在里面可统一定义空间数 据的工作及对应形式; 不论是空间还是非空间的数据资源 都可以进行相应治理, 这使得为制作研究统一空间数据库 系统制造了一个十分有利的环境。

\section{4 电力体系的安全及平稳性能}

在我国电力体系对于经济的发展起着至关重要的作用, 作为一个实施工作体系, 是否平稳安全是它一定要思考的。 而对于它的安全和平稳性我们要从电力设施的工作、相关 数值、网络运行等这些来进行着手。前提是系统本身的稳 固, 也就是体系要具有多种形式规格的相应数据和针对对 象运行平稳的优点, 迅速且强大的复原体系; 对于多用户同 时访问运行系统是否稳定、快速、正常, 能不能双机热备份, 这些都需要我们进行思考。

\section{5 结束语}

综上所说, 对于电力体系的现代化就是使用电脑网络 的相关技术来完成对电力体系的全方位控制, 这其中包含: 实际工作的总线控制配置、光传输并运用两种以上的处理 计算方式技术、不同于传统对象数据库的被动形式而产生 的主动式技术。而其技术含义就是为了能让电力的界限可 以变得越来越大, 进而提高对电的供应实力, 以及电力的稳 定安全性能, 从而让电力体系可能适用、安全的进行, 进而 让我国电力事业可以长久发展下去。

\section{[参考文献]}

[1]胡君君.电力系统及其自动化技术的应用探讨[J].机 电信息,2011(12):53+58.

[2]朱淋, 徐秀英, 肖中图.浅论电力系统及其自动化技术 的应用能力 $[\mathrm{J}]$.科技风,2010(04):63.

[3]洪秀平. 浅论电力系统及其自动化技术的应用能力 [J].科技致富向导,2010(05):36. 